\title{
Additive effect of dabigatran and high-dose aspirin in the development of haemorrhagic pleural effusion in a patient with tuberculous pleuritis Duygu Acar Karagül ${ }^{1}$, Veysel Özgür Barış ${ }^{2}$, Demet Karnak ${ }^{1}$
}

1. Chest Disease Department, Ankara University Medical Faculty, Ankara, Turkey

2. Cardiology Department, Ankara University Medical Faculty, Ankara, Turkey

\begin{abstract}
Tuberculous pleuritis can rarely cause haemorrhagic pleural effusion. Dabigatran etexilate can have an additive effect on increasing the risk of haemorrhage. Aspirin cannot cause major haemorrhage, but in the elderly it can cause gastrointestinal bleeding via ulceration of the gastrointestinal mucosa. We report here the case of a 77-year-old male who presented to the hospital with a 2-month history of progressive dyspnoea. He had been taking dabigatran etexilate $(220 \mathrm{mg}$ ) and high-dose acetylsalicylic acid (aspirin; $300 \mathrm{mg})$ daily for chronic atrial fibrillation. A chest X-ray revealed a moderately sized right pleural effusion confirmed by a computed tomography scan, which also showed bronchiectasis of both lungs. Dabigatran was discontinued and aspirin was decreased to the minimal therapeutic dose of $100 \mathrm{mg}$ before thoracentesis was performed. Lymphocyte-predominant (50\%) haemorrhagic fluid of $500 \mathrm{ml}$ was drained, positive for acid-fast bacilli smear and polymerase chain reaction of Mycobacterium tuberculosis. A chest tube was placed and an additional $1250 \mathrm{ml}$ of haemorrhagic exudate drained out. We treated the patient with a routine regimen of antituberculous medication and the infection resolved without complications other than the bronchiectasis present before treatment. We think that the combination of dabigatran etexilate and high doses of aspirin increased the risk of pleural haemorrhage in this patient with tuberculous pleuritis.
\end{abstract}

Key Words: Aspirin, dabigatran etexilate, pleural haemorrhagic effusion, tuberculous pleuritis

\section{Case report}

A 77-year-old male non-smoker presented to the outpatient unit of the Chest Disease Department at Ankara University Hospital with a 2-month history of progressive dyspnoea in September 2014. His past medical history was significant for atrial fibrillation, diabetes mellitus type 2, coronary artery disease, hypertension, hyperlipidaemia, and congestive heart failure. He had taken dabigatran etexilate $110 \mathrm{mg}$ orally twice daily for the previous 2 months. His other medication consisted of metoprolol $50 \mathrm{mg} /$ day, atorvastatin $20 \mathrm{mg} /$ day, metformin $1000 \mathrm{mg}$ twice daily, and acetylsalicylic acid (aspirin) $300 \mathrm{mg} /$ day. The patient's vital signs were normal except for an irregular pulse rate of 130 beats $/ \mathrm{min}$. His body mass index (BMI) was $17.5 \mathrm{~kg} / \mathrm{m}^{2}$. He had decreased breath sounds in the right lower lung field, with dullness to percussion and bilateral coarse crackles scattered throughout the lung fields.

An electrocardiogram showed atrial fibrillation. On admission his white blood cell count was $9.2 \times 10^{3} / \mathrm{mm}^{3}$ (normal range: $4.0-12.4 \times 10^{3}$ ), haemoglobin was $12.7 \mathrm{~g} /$ dl (normal range: 11.6-15.2), platelet count was $295 \times 10^{3} /$ $\mathrm{mm}^{3}$ (normal range: $141-320 \times 10^{3}$ ), haematocrit was $37 \%$ (normal range: 41-50\%), blood glucose was $115 \mathrm{mg} / \mathrm{dl}$ (normal range: 100-110), blood urea nitrogen was $19 \mathrm{mg} / \mathrm{dl}$ (normal range: $7-20$ ), serum creatinine $0.84 \mathrm{mg} / \mathrm{dl}$ (normal range: $0.7-1.2$ ) with an estimated creatinine clearance of 89 $\mathrm{ml} / \mathrm{min}$ (using the Modification of Diet in Renal Disease equation), the prothrombin time (PT) was $20 \mathrm{~s}$ (normal range: 11-13) and activated partial thromboplastin time (aPTT) was $52.2 \mathrm{~s}$ (normal range: $25-36$ ) with international normalised ratio (INR) level of 2.6 (normal range: 1-1.3). A chest $\mathrm{X}$-ray revealed a moderate right pleural effusion (Figure 1A). A chest computed tomogram showed right pleural thickening and bilateral lower lobe bronchiectasis
(Figure 1B). Echocardiography revealed left ventricular systolic dysfunction with a $45 \%$ ejection fraction.

Aspirin level was reduced to $100 \mathrm{mg} /$ day, a safe limit for bleeding risk, and dabigatran etexilate was discontinued for 3 days to decrease PT and aPT'T levels and INR within the normal range (12 s, $35 \mathrm{~s}$ and 1.3, respectively). Flexible bronchoscopy (FB) was performed revealing coarse pleated bronchial mucosa, haemorrhage with FB touch, and smooth outer compression at the right lower lobe. Haemorrhagic fluid (500 ml) was initially sampled by thoracentesis from the right pleural space (Figure 1C,D). In the same session, using ultrasound guidance a chest tube was placed to drain all the haemorrhagic material, along with a pleural biopsy revealing granulomatous inflammation. Within $48 \mathrm{~h}, 1250 \mathrm{ml}$ of haemorrhagic accumulation was seen on the drainage system until it was removed (Figure 1E). Cytological examination revealed excessive erythrocytes and lymphocytes (50\% excess), but no malignant cells were seen. The haematocrit was $18.5 \%$, and there was no growth of microorganisms on common cultures.

As the diagnosis of tuberculous pleuritis (TP) was proven via smear and polymerase chain reaction (PCR) positivity for acid-fast bacilli of the fluid, four-drug oral antituberculous therapy including rifampicin, isoniazid, ethambutol, and pyrazinamide was commenced at the fifth hospital day and was well tolerated. Prophylactic bemiparin sodium 5000 IU/day subcutaneously was given until the end of the treatment instead of oral anticoagulants to prevent probable thrombosis.

Dyspnoea was resolved in the first hospital week and the patient was discharged on the 10th hospital day, with only right costophrenic angle blunting without any recurrence. Antituberculous therapy was continued for 6 months. He stayed alive for 5 years then passed away from cardiac reasons. 


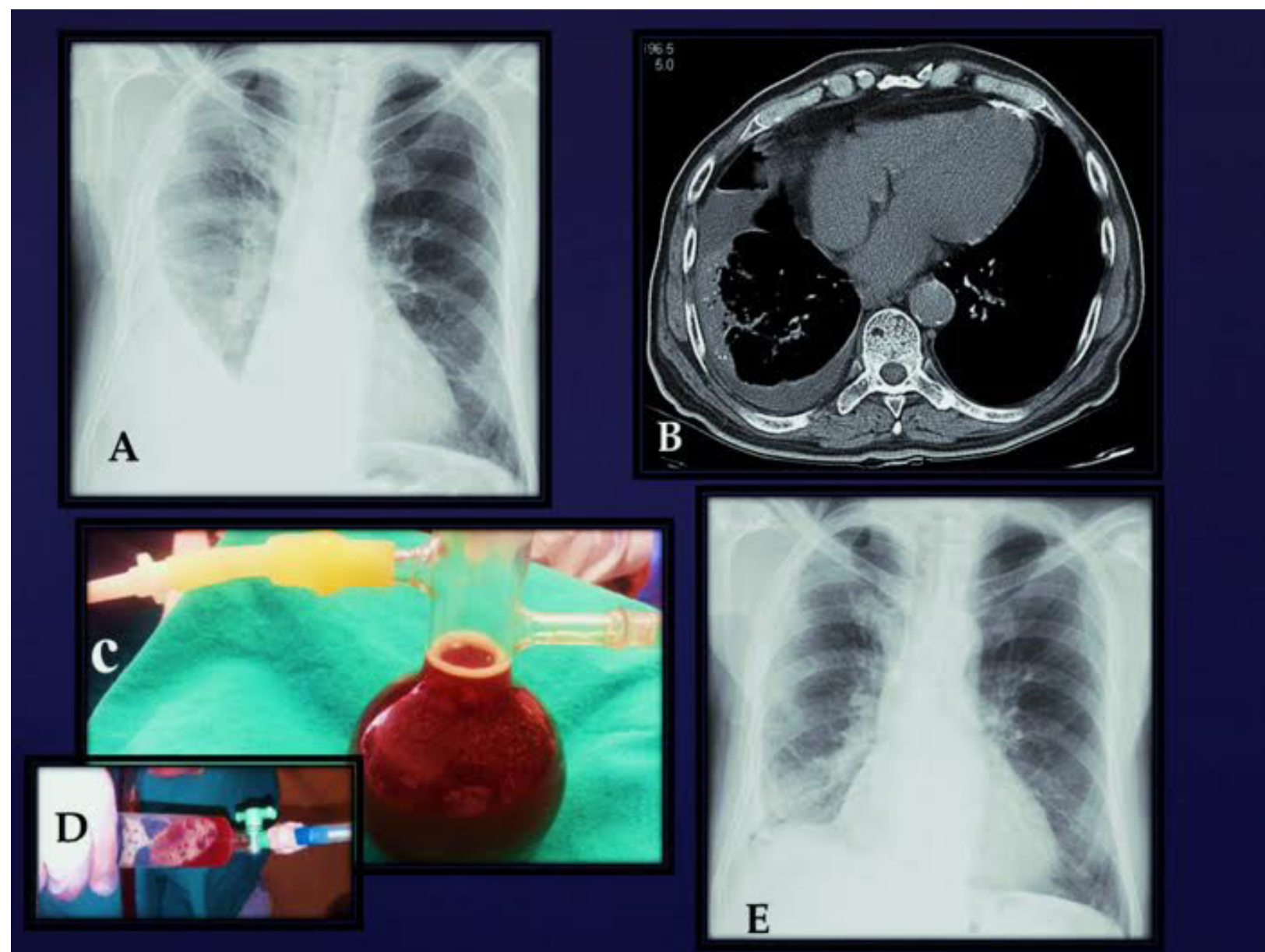

Figure 1. (A) Chest X-ray with right-sided pleural fluid. (B) Thorax computed tomogram showed pleural fluid and bronchiectasis on the right lower lobe. (C,D) Haemorrhagic pleural fluid seen during aspiration. (E) Chest $X$-ray after drainage of the fluid.

\section{Discussion}

Exudative pleural effusion with pleural lymphocytosis (suggestive for tuberculosis), acid-fast bacilli stain, PCR evaluation, and histopathological examination led us to a definitive diagnosis of TP. As haemorrhagic effusion is known as unlikely in TP in clinical practice ${ }^{1}$, the cause of a higher amount of pleural haemorrhagic effusion was needed to be explained at the first stage in this patient.

First, the patient was taking two major drugs that could contribute to haemorrhage, aspirin (300 $\mathrm{mg} /$ day) and dabigatran etexilate $(110 \mathrm{mg}$, twice daily). Although aspirin can act as a prostaglandin synthetase inhibitor blocking aggregation of the platelets, it is not recorded as a causative agent of pleural haemorrhagic effusion by itself at any dose in the literature in English ${ }^{2}$. Furthermore, its cessation is not recommended before bronchoscopy as it cannot increase the risk of bleeding ${ }^{3,4}$. However, a higher dose $(300 \mathrm{mg})$ of aspirin can cause or contribute to major bleeding occurring first in the gastrointestinal system (GIS) followed by the cerebrovascular system ${ }^{5}$. In this case, high-dose aspirin may have contributed to the accumulation of haemorrhagic effusion, but if so, there should also have been bleeding in the GIS or cerebrovascular system in addition to the pleural space. Therefore, the absence of these effects suggested that this drug might not be the only cause of haemorrhage. The aspirin dose was reduced to $100 \mathrm{mg}$ before the procedures and no fresh haemorrhage related to these procedures was observed.

Second, the patient was given dabigatran etexilate as the only oral antithrombin agent, which is one of the direct factor
$\mathrm{Xa}$ inhibitors that prevents prothrombin from cleaving to thrombin. It binds directly to factor $\mathrm{Xa}$, rather than enhancing the activity of antithrombin, as is done by heparin. Acute bleeding episodes, such as intracranial haemorrhage, haemopericardium, and diffuse alveolar haemorrhage have been reported to be associated with or worsening from use of dabigatran etexilate ${ }^{6}$. Two similar cases with nonvalvular atrial fibrillation have been reported in patients of 72 and 83 years of age who developed haemothorax and were receiving $110 \mathrm{mg}$ of dabigatran twice daily. These patients were managed by cessation of the drug and a large amount of chest drainage (1400 and $2500 \mathrm{ml}$, respectively). It is claimed that age or renal impairment could play some role in those with increased bleeding risk ${ }^{6}$. Therefore, it has been suggested that plasma levels should be checked and the dose should be individualized according to the plasma level, otherwise, there is a risk of complication with bleeding 15\% higher than the normal population.

Combining aspirin with novel anticoagulants does not provide additional benefit in stroke reduction and is associated with an increased risk of bleeding complications. Therefore, care should be taken to determine if and when the benefits of concomitant aspirin outweigh the risks in patients with atrial fibrillation already on oral anticoagulants ${ }^{7}$. In a large study, high dose $(300 \mathrm{mg}$ ) of aspirin combined with higher doses (300 $\mathrm{mg}$ ) of dabigatran can contribute to major bleeding events. However, lower doses (50-150 mg) of dabigatran cannot prevent thromboembolic episodes even with aspirin ${ }^{8}$. We think that in this case, adding dabigatran in the course of aspirin can be a major contributing factor when taking into account prolonged PT, aPT'T, and INR which were hardly 
becoming normal in 3 days of cessation. Since dabigatran with a long half-life (12-17 h) is given twice daily in advanced patient age, plasma accumulation may cause haemothorax despite borderline-normal kidney functions.

This patient was old, had diabetes, and was malnourished (low BMI). These are all risk factors for active tuberculosis disease. TP can be seen with or without pulmonary parenchymal tuberculosis. In this patient, there was no finding of tuberculosis affecting pulmonary parenchymal tissue reflecting active disease. However, pleural effusion was high, causing dyspnoea. Pleural fluid in TP is usually straw-coloured exudate, or rarely slightly bloody, usually without exceeding one- to two-thirds of the hemithorax? On the contrary, several haemorrhagic types have been reported in the literature ${ }^{10-12}$. In a case report, it was stated massive haemorrhagic pleural effusion was extremely rare. Pleural biopsy has the best diagnostic yield for TP and as it was defined as a protean disease (having a thousand faces), it should always be in the differential diagnosis of a patient with a lymphocytic pleural exudate, whether it is haemorrhagic or not, small or massive ${ }^{10}$. In large reviews, TP was complicated with empyema, fibrothorax, chylothorax, and other possibilities; however, haemorrhagic effusion can be described in only $11 \%{ }^{11,12}$. In a study, only $9 \%$ of the patients $(n=100)$ had haemorrhagic effusion whereas $90 \%$ had straw-coloured effusion ${ }^{13}$. Therefore, it is imperative to investigate drugs that contribute to haemorrhagic effusion.

Pleural drainage was also recommended to relieve dyspnoea and avoid complications ${ }^{8-12}$. In this case, the fluid probably exceeded two-thirds of the hemithorax and/or would become complicated had it not been drained.

To conclude, physicians should be aware of the antiaggregant/anticoagulant drugs used by the patient or infectious disease such as tuberculosis, especially in cases with pleural haemorrhagic effusion.

\section{References}

1. Mandovra N, Vaidya PJ, Chhajed PN. Current approaches to tuberculous pleural effusion. Astrocyte. 2017;4(2):87-93. https://doi. org/10.4103/astrocyte.astrocyte_59_17.

2. Serebruany VS, Steinhubl SR, Berger PB, Malinin AU, Baggish JS, Deepak LB, et al. Analysis of risk of bleeding complications after different doses of aspirin in 192,036 patients enrolled in 31 randomized controlled trials. Am J Cardiol. 2005;95(10):1218-22. https://doi. org/10.1016/j.amjcard.2005.01.049.
3. Kupeli E, Karnak D, Mehta AC: Flexible bronchoscopy. In: Mason RJ, Broaddus VC, Martin TR, King TE, Schraufnagel DE, Murray JF, Nadel JA, editors. Textbook of respiratory medicine. 5th ed. Philadelphia, Volume 1. Saunders Elsevier; 2010. p. 490.

4. Du Rand IA, Blaikley J, Booton R, Chaudhuri N, Gupta V, Khalid $\mathrm{S}$, et al. British Thoracic Society Bronchoscopy Guideline Group. Summary of the British Thoracic Society guideline for diagnostic flexible bronchoscopy in adults. Thorax. 2013; 68(8):786-7. https://doi. org/10.1136/thoraxjnl-2013-203629.

5. Sugawara M, Goto Y, Yamazaki T, Teramoto T, Oikawa S, Shimada K, et al. Japanese Primary Prevention Project (JPPP) Study Group. Low-dose aspirin for primary prevention of cardiovascular events in elderly Japanese patients with atherosclerotic risk factors: subanalysis of a randomized clinical trial (JPPP-70). Am J Cardiovasc Drugs. 2019;19(3):299-311. https://doi.org/10.1007/s40256-018-0313-0.

6. Cano EL, Miyares MA. Clinical challenges in a patient with dabigatran-induced fatal hemorrhage. Am J Geriatr Pharmacother. 2012;10(2):160-3. https://doi.org/10.1016/j.amjopharm.2012.02.004.

7. Hernandez I, Baik SH, Piñera A, Zhang Y. Risk of bleeding with dabigatranin atrial fibrillation. JAMA Intern Med. 2015;175(1):18-24

8. Ezekowitz MD, Reilly PA, Nehmiz G, Simmers TA, Nagarakanti R, Parcham-Azad K, et al. Dabigatran with or without concomitant aspirin compared with warfarin alone in patients with nonvalvular atrial fibrillation (PETRO Study). Am J Cardiol. 2007;100(9):1419-26. https://doi.org/10.1016/j.amjcard.2007.06.034.

9. Shaw JA, Diacron AH, Koegelenberg CFN. Tuberculous Pleural effusion. Respirology. 2019;24(10):962-71. https://doi.org/10.1111/ resp. 13673.

10. Mermigkis C, Kopanakis A, Psathakis K, Karagiannidis N, Kastanakis M, Patentalakis M, et al. A massive hemorrhagic pleural effusion does not exclude the diagnosis of tuberculosis: a case report. Cases J. 2009;2:8707. https://doi.org/10.4076/1757-1626-2-8707.

11. Jolobe OMP. Atypical pleural effusion. Eur J Intern Med. 2011;22(5):456-9. https://doi.org/1016/j.ejim.2011.03.011.

12. Antonangelo L, Vargas FS, Seiscento M, Bombarda S, Teixeira L, Sales RKB. Clinical and laboratory parameters in the differential diagnosis of pleural effusion secondary to tuberculosis or cancer. Clinics. 2007;62(5):585-90. https://doi.org/10.1590/s180759322007000500009 .

13. Ibrahim WH, Ghadban W, Khinji A, Yasin R, Soub H, Al-Khal AL, et al. Does pleural tuberculosis disease pattern differ among developed and developing countries? Respir Med. 2005;99(8):1038-45. https:// doi.org/10.1016/j.rmed.2004.12.012. 


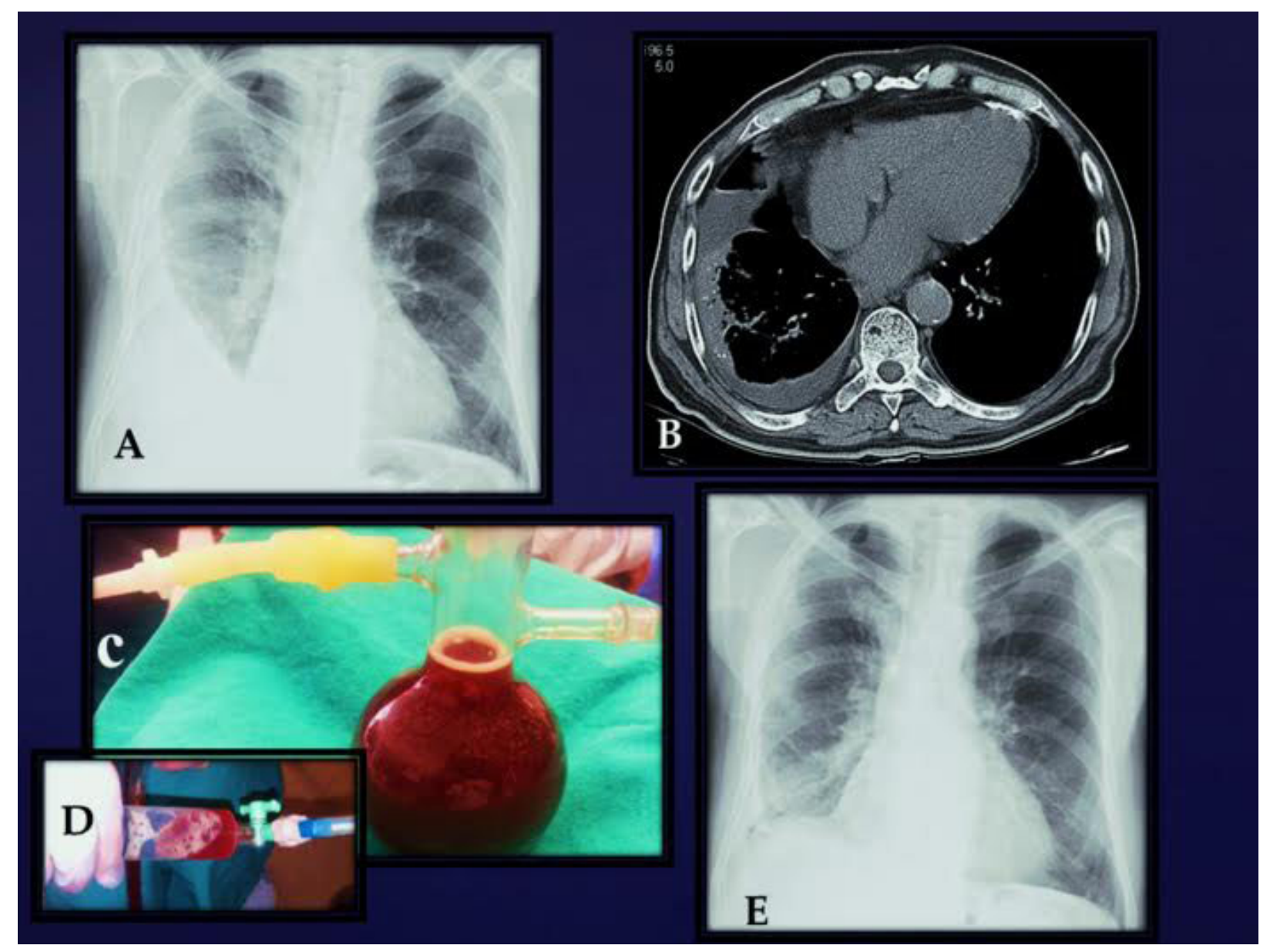

